Article

\title{
Structural and Molecular Characterization of meso-Substituted Zinc Porphyrins: A DFT Supported Study
}

\section{Rudolf Slota $^{1, *}$, Malgorzata A. Broda ${ }^{1}$, Gabriela Dyrda ${ }^{1}$, Krzysztof Ejsmont $^{1}$ and} Giuseppe Mele ${ }^{2}$

1 Faculty of Chemistry, Opole University, ul. Oleska 48, 45-052 Opole, Poland

2 Department of Engineering and Innovation, University of Salento, Via Arnesano, 73100 Lecce, Italy

* Author to whom correspondence should be addressed; E-Mail: rslota@uni.opole.pl;

Tel.: +48-77-452-7131; Fax: +48-77-452-7101.

Received: 2 November 2011; in revised form: 22 November 2011 / Accepted: 22 November 2011 / Published: 1 December 2011

\begin{abstract}
Structural parameters of a range of over 100 meso-substituted zinc porphyrins were reviewed and compared to show how far the nature of the functional group may affect the interatomic distances and bond angles within the porphyrin core. It was proved that even despite evident deformations of the molecular structure, involving twisting of the porphyrin's central plane, the coupled $\pi$-bonding system remains flexible and stable. DFT calculations were applied to a number of selected porphyrins representative for the reviewed compounds to emphasize the relevance of theoretical methods in structural investigations of complex macrocyclic molecular systems. Experimental and DFT-simulated IR spectral data were reported and analyzed in context of the individual molecular features introduced by the meso substituents into the porphyrin moiety base. Raw experimental spectral data, including ${ }^{1} \mathrm{H}$ - and ${ }^{13} \mathrm{C}-\mathrm{NMR}$, UV-Vis, FTIR, XRD, and other relevant physicochemical details have been provided for a specially chosen reference zinc porphyrin functionalized by tert-butylphenyl groups.
\end{abstract}

Keywords: zinc porphyrins; molecular structure; DFT theoretical calculations; IR spectra 


\section{Introduction}

The incessant scientific interest in porphyrins finds its origin in their affinity with chlorophyll and heme, the Nature-created metalloporphyrin species considered crucial to evolution of life on Earth. Hence, synthetic analogues featuring the characteristic porphyrin macrocycle have been expected to reveal a great application potential. Relative easy synthesis at moderate cost and a world of possibilities in creating of diverse structural modifications offer almost unlimited prospects for molecular design. The most important properties of the porphyrins are manifested first in their electronic absorption spectra, reflecting the unusual physicochemical nature of this peculiar class of compounds. Nevertheless, it is the character of the bonding setup which determines the specific chemistry of the porphyrin family. A comprehensive compilation entirely devoted to porphyrins can be found in the Handbook of Porphyrin Science [1].

In general, the chemistry of different metalloporphyrins is controlled by the complexed metal and the kind of peripherally and/or axially fixed substituents. These factors influence the electronic density distribution within the core of the macrocycle and thus determine its reactivity and stability, as well. Structural details are essential in learning the basic chemistry of such compounds. Therefore, crystallographic and molecular studies are of principal importance in the search for their unique properties.

Natural porphyrins, e.g., the chlorophylls, have been substituted predominantly at the pyrrole periphery. Undoubtedly, the four carbon atoms (meso carbons) coupling the pyrrole units to form the characteristic macrocycle represent the weakest sites of the whole bonding system (Figure 1). They seem to be an easy target for a chemical attack particularly when reactive electron acceptors are present in the ambient. However, when substituted by bulky particles that could provide some kind of shielding, the strength of the porphyrin moiety may increase. Such structurally induced reinforcement is reflected, e.g., in considerably higher thermal stability of meso-substituted synthetic porphyrins [2,3], compared to the chlorophylls and similar analogues [4]. Depending on the substituents, the resulting structure of the porphyrin core may be more or less affected, thus making the compound more or less sensitive to the influence of the environment. Phenyl-derived radicals seem to be appropriate candidates for protecting the bonding set-up at the meso sites. Therefore, a wide range of them will be discussed below in the context of their potential influence upon the structural parameters of the porphyrin moiety.

Porphyrins with all meso carbons equally functionalized by substituted phenyls belong to the most extensively explored ones within the last few decades and still remain an attractive research subject. Among them, those including the tert-butylphenyl groups have gained special attention (Figure 2). Particularly, they were found to demonstrate very interesting photochemistry, which is believed fundamental for the development of novel application areas. For instance, the $\mathrm{Cu}(\mathrm{II})$ porphyrin with tert-butylphenyl groups in either of the meso positions, CuTBPP, (where TPBP denotes the 5,10,15,20tetrakis(4-tert-butylphenyl) porphyrin moiety, $\mathrm{C}_{60} \mathrm{H}_{60} \mathrm{~N}_{4}$ ) revealed reasonable photocatalytic activity in composite sensitizer- $\mathrm{TiO}_{2}$ catalyst systems [5,6]. The identically functionalized ZnTBPP complex [7], which in the present work has been chosen as a reference model compound, is supposed to feature similar chemistry. Moreover, a range of TBPP metalloporphyrins revealed lipophilic properties and displayed diverse activity when embedded into liposome membranes [8]. This fact may be crucial to their potential application in biological systems. 
Figure 1. General structure of the tetra-meso-substituted Zn-porphyrins (OMe = methoxy; $\mathrm{tBu}=$ tert-butyl).
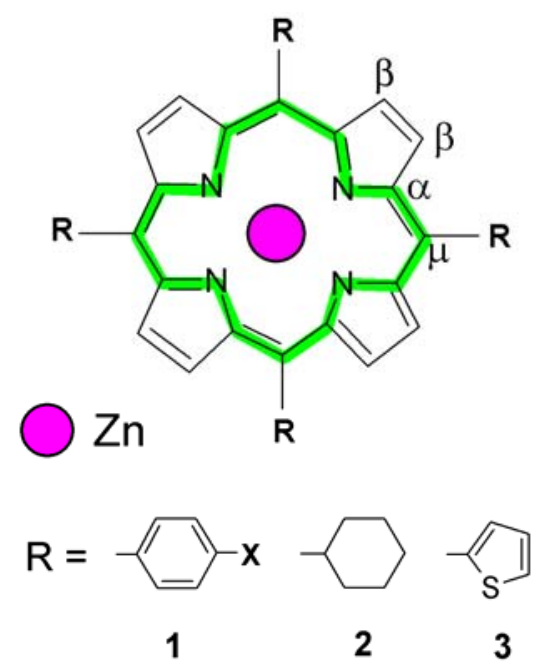

$\mathrm{X}=\mathrm{H}, \mathrm{F}, \mathrm{OH}, \mathrm{CN}, \mathrm{OMe}, \mathrm{tBu}$

Figure 2. Molecular structure of diverse Zn-pophyrins (derived from crystallographic data). Structures (a) and (b) refer to ZnTBPP [7]; (c) Cyanophenyl analogue ( $\mathrm{R}=1$, $\mathrm{X}=\mathrm{CN})$ [9]; (d) Cyclohexyl analogue $(\mathrm{R}=2)$ [10]; (e) Bent pyrrole units of the porphyrin core, adapted from [11]; two opposite nitrogen atoms have been highlighted as blue balls to emphasize the distortion.

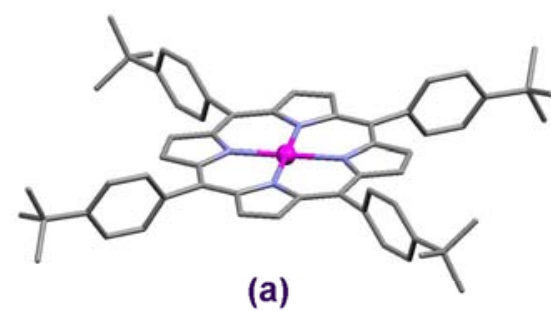

(a)

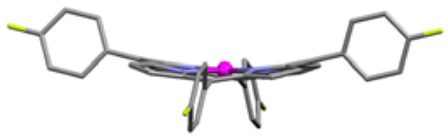

(c)

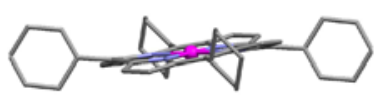

(d)

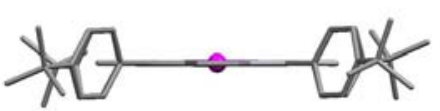

(b)

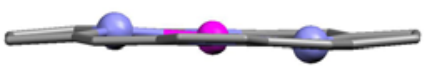

(e)

Undoubtedly, structure-related chemical properties and the morphology of the crystalline solids are important factors, which may influence the behavior of a porphyrin-based system under specified circumstances considerably. In the present study over 100 crystal structures of Zn-porphyrins including diverse phenyl-based groups linked to the macrocycle via the meso carbons have been compared with each other. Particularly, the geometry of the bonding system within the porphyrin core was analyzed to find out how far this can be modified by the meso-substituents itself and/or the solvent species incorporated into the crystal lattice. This study was supplemented by theoretical molecular modeling and had been expected to provide a more profound insight into the nature of meso-substituted porphyrins. During the recent years DFT calculations became an important tool in analyzing structural and spectral properties even in the case of complex molecular systems like those represented by the 
porphyrin species [12-14]. Based on our previous experience [15], we have extended the theoretical approach by simulating the IR vibrational spectra for selected compounds, representative for the analyzed structures. In this way, to some extent, it was possible also to estimate the impact of the particular meso-functionalization on the dynamic behavior of the whole macrocyclic setup. Moreover, by comparing the experimental and DFT-derived results we intended to highlight the growing significance of theoretical calculations in contemporary physicochemical studies.

\section{Results and Discussion}

For the reason of this work, we chose a reference model porphyrin 5,10,15,20-tetrakis(4-tertbutylphenyl)porphyrinato zinc(II) (ZnTBPP), presented in Figure $1(\mathrm{R}=1, \mathrm{X}=t$-Bu) and Figure 2. This particular compound was extensively studied by our group for its crystallographic structure and diverse physicochemical properties [7,8]. The ZnTBPP complex has been characterized in the Experimental section; however XRD powder diffraction records along with raw spectral data have been reported in the Supplementary Information appendix. From the single crystal measurements it has followed that the model compound ZnTBPP represents a very well established molecular system. The $\mathrm{Zn}$ atom is found in a plane defined by the four pyrrole-nitrogen atoms and the N-Zn-N bond angles are ca. $90^{\circ}$, showing ideal square-planar coordination. The macrocycle itself shows no apparent distortion and one may consider it as "perfectly flat" (Figure 2b). The meso substituted benzene rings are not perpendicular to the plane of the macrocycle, yielding dihedral angles of $78.4^{\circ}$ and $79.2^{\circ}$. Average bond lengths related to the porphyrin macrocycle have been collected in Table 1. For a complete listing of bond distances and angles see the previous work [7]. This model structure was compared with crystal data acquired for a set of 105 selected structural analogues, derived from a crystallographic database available elsewhere [16-19] and corresponding to those shown in Figure 1 for $\mathrm{R}=1$ (i.e., various phenyl-based groups); the relevant structural data have been collected in Supplementary information appendix. The study was principally dedicated to the influence of diverse meso substituents on the geometrical relations within the Zn-porphyrin macrocycle and essentially it was focused on the bonds constituting the inner core, i.e., the coupled $\pi$-electronic system. For the selection of molecules as listed in Figure 1, the molecular structure was obtained also from DFT theoretical calculations. Versatile possibilities offered by the Gaussian 09 package [20] prompted us to calculate the IR spectra of these compounds and analyze the effect of the particular substituents on the frequency of bond oscillations within the porphyrin core. Moreover, animation of the individual vibrational modes simulating the dynamic behavior of the porphyrin moiety allowed relating it with the potential effect of the substituting groups, thus supplementing the results of structural investigations.

\subsection{Molecular Effects in Real Crystals and DFT-Derived Zn-Porphyrins}

Generally, the X-ray determined structure of ZnTBPP [7] fits well into the range of parameters previously reported for related Zn-porphyrins of similar molecular construction [16-19]. In addition, the values following from the theoretical model very well agree with the measured data, as reported in Tables 1 and 2. This is an important result illustrating the significance and credibility of theoretical modeling in advanced molecular studies of porphyrins. 
Table 1. Average interatomic distances within the pyrrole subunit, meso-bridges and $\mathrm{Zn}-\mathrm{N}$ for the model compound ZnTBPP $(\mathrm{R}=1, \mathrm{X}=\mathrm{tBu})$ and diverse zinc porphyrin molecules (acc. to Figure 1); "crystal" applies to values calculated from real crystallographic data reported elsewhere and "DFT" refers to theoretical results obtained in this work. For R $=1$ the data represent average values of 105 diverse molecules analyzed.

\begin{tabular}{ccccccc}
\hline Porphyrin type & & $\mathbf{Z n}-\mathbf{N}$ & $\mathbf{C}_{\boldsymbol{\alpha}}-\mathbf{N}$ & $\mathbf{C}_{\boldsymbol{\alpha}}-\mathbf{C}_{\boldsymbol{\beta}}$ & $\mathbf{C}_{\boldsymbol{\beta}}-\mathbf{C}_{\boldsymbol{\beta}}$ & $\mathbf{C}_{\boldsymbol{\alpha}}-\mathbf{C}_{\boldsymbol{\mu}}$ \\
\hline ZnTBPP (crystal) & {$[7]$} & 2.04 & 1.38 & 1.44 & 1.36 & 1.40 \\
ZnTBPP (DFT) & & 2.07 & 1.40 & 1.46 & 1.37 & 1.42 \\
$\mathrm{R}=\mathbf{1}$ (crystal) & {$[16,17]$} & 2.04 & 1.38 & 1.44 & 1.35 & 1.40 \\
$\mathrm{R}=\mathbf{2}$ (crystal) & {$[10,21]$} & 2.03 & 1.38 & 1.44 & 1.35 & 1.40 \\
$\mathrm{R}=\mathbf{3}$ (crystal) & {$[11]$} & 2.02 & 1.36 & 1.43 & 1.33 & 1.38 \\
\hline
\end{tabular}

Table 2. Core perimeter and average $\mathrm{C}_{\mu}-\mathrm{R}$ distance in selected tetra-meso-substituted zinc porphyrins: Experimental (exp.) and DFT-derived results (all values in $\AA$ ); porphyrin structures according to Figure 1; references to literature apply exclusively to experimental data.

\begin{tabular}{cccccc}
\hline Porphyrin type & References & $\begin{array}{c}\text { Core } \\
\text { perimeter } \\
\text { (exp.) }\end{array}$ & $\begin{array}{c}\text { Core } \\
\text { perimeter } \\
\text { (DFT) }\end{array}$ & $\begin{array}{c}\mathbf{C}_{\boldsymbol{\mu}}-\mathbf{R} \\
\text { distance } \\
\text { (exp.) }\end{array}$ & $\begin{array}{c}\mathbf{C}_{\boldsymbol{\mu}}-\mathbf{R} \\
\text { distance } \\
\text { (DFT) }\end{array}$ \\
\hline $\mathrm{R}=\mathrm{H}$ & & $-{ }^{1)}$ & 22.37 & $-{ }^{1)}$ & 1.09 \\
$\mathrm{R}=\mathbf{1}, \mathrm{X}=\mathrm{H}$ & {$[22]$} & 22.22 & 22.50 & 1.50 & 1.50 \\
$\mathrm{R}=\mathbf{1}, \mathrm{X}=\mathrm{F}$ & {$[23]$} & 22.14 & 22.49 & 1.48 & 1.50 \\
$\mathrm{R}=\mathbf{1}, \mathrm{X}=\mathrm{OH}$ & {$[24]$} & 22.24 & 22.50 & 1.50 & 1.50 \\
$\mathrm{R}=\mathbf{1}, \mathrm{X}=\mathrm{CN}$ & {$[9]$} & 22.24 & 22.49 & 1.50 & 1.50 \\
$\mathrm{R}=\mathbf{1}, \mathrm{X}=\mathrm{OMe}$ & {$[25]$} & 22.28 & 22.50 & 1.51 & 1.50 \\
${ }^{2)} \mathrm{R}=\mathbf{1}, \mathrm{X}=\mathrm{tBu}$ & {$[7]$} & 22.24 & 22.50 & 1.50 & 1.50 \\
$\mathrm{R}=\mathbf{2}$ & {$[21]$} & 22.29 & 22.57 & 1.53 & 1.54 \\
$\mathrm{R}=\mathbf{3}$ & {$[11]$} & 21.99 & 22.49 & 1.47 & 1.49 \\
\hline & ${ }^{1)}$ crystal data not available; ${ }^{2)}$ ZnTBPP. & &
\end{tabular}

Comprehensive analysis of available crystallographic data supported by a detailed literature study have proved that the ideal molecular symmetry, as observed in our ZnTBPP complex, may be more or less distorted in the case of diverse tetra-meso-substituted Zn-porphyrins reflecting the impact of the incorporated species and when crystallized from different solvents (Figure 2). Structures with the bent pyrrole rings are produced, e.g., when $\mathrm{X}=\mathrm{CN}$ [9] or F [23], Figure 2c. Such "saddle" conformations usually result from interactions between the adjacent macrocycles within the crystal lattice, often involving the solvent species (solvent effect). This is particularly pronounced when bulky solvent molecules have been accommodated by the crystal unit, as found, e.g., in the case of a Zn-porphyin clathrate $(\mathrm{R}=\mathbf{1}, \mathrm{X}=\mathrm{Br})$ containing 4 mesithylene species per one of the complex [26]. Another interesting example is the pentakis-guaiacol clathrate $(\mathrm{R}=\mathbf{1}, \mathrm{X}=\mathrm{OH})$ characterized by a distinct discrepancy in interatomic distances, $5 \%-10 \%$ for the same bond type [24]. Oxygen- and/or chloridecontaining solvents seem to produce structural disorder particularly for porphyrins substituted by $\mathrm{R}=1$ with $X \neq H$, although some slight deviations may be observed even when $X=H$ [22]. In the case of 
methoxybenzene solvates, the oxygen atom of the "methoxy" group was found to interact with the $\mathrm{Zn}$ atom slightly pulling it out from the plane defined by the four pyrrole nitrogens $(R=1, X=F)[23]$, showing up a convex-shaped porphyrin core, unique among the other structures reviewed of the Cambridge Structural Database [16,17]. The bent pyrroles and bond length differences of 3\%-5\% have also been exhibited in molecules where $\mathrm{R}=1$ and $\mathrm{X}=n$-octyl [27]. In this case the structural deviations have been generated presumably by the aliphatic substituents. Nevertheless, in each of these "distorted" molecules, the core perimeter was found close to $22.24 \AA$, i.e., well within the range determined for all structures regarded, Table 2.

Additionally, the interatomic distances within Zn-porphyrins including $\mathrm{R}=\mathbf{1}$ have been compared with those involving $\mathrm{R}=\mathbf{2}$ and $\mathbf{3}$, because of diverse volume of these meso-coupled species relative to $\mathrm{R}=\mathbf{1}$. The cyclohexyl radical $(\mathrm{R}=\mathbf{2},[10])$ does not noticeable influence the size of the porphyrin core, although the macrocycle itself proves slightly twisted and the meso $\mathrm{C}_{\mu}-\mathrm{R}$ bonds appear somehow longer ( $2 \%$ ) than in the case of $\mathrm{R}=\mathbf{1}$ (Figure $2 \mathrm{~d}$, Table 2). Almost flat macrocycle and bond lengths typical for the studied Zn-porphyrin family are demonstrated also for $\mathrm{R}=\mathbf{3}$ [11]. However, in this case the pyrroles of the porphyrin core are slightly bent, as shown in Figure 2e, and this type of distortion was relative frequently observed among the reviewed $\mathrm{Zn}$-porphyrins. The $\mathrm{C}_{\mu}-\mathrm{R}$ distances are ca. $2 \%$ shorter than for ZnTBPP, because of the smaller volume of the thienyl radical. In fact, for all of the molecules considered in this survey, the average $\mathrm{C}_{\mu}-\mathrm{R}$ distance is kept relative close to $1.50 \AA$. Interestingly, for another bulky substituent, 3,5-di-tert-butylphenyl, the porphyrin core proved ideal planarity, despite considerable torsion of the $\mathrm{C}_{\mu}-\mathrm{R}$ bonds (ca. $64^{\circ}$ ) and some differences in length (ca. 4\%) of the $\mathrm{C}_{\beta}-\mathrm{C}_{\beta}$ periphery within the pyrrole units [28].

The existence of a very stable molecular core is a crucial conclusion to this analysis of diverse meso-substituted Zn-porphyrin structures. Regardless of R and X (Figure 1), there is no such tendency revealed, that the bonds at the meso carbons be considerable affected. In most of the analyzed structures [16,17], even when the pyrroles were bent or twisted, the C-N and C-C bonds constituting the coupled $\pi$-electronic system are supposed to retain their stability since they do not differ from each other for more than $2 \%$ in length. Indeed, this fact has been confirmed by the crystallographic data reported thus far and reflected in a very similar porphyrin core perimeter displayed by the compounds compared, as shown in Table 2. This resulted also from DFT calculations, and the theoretically derived porphyrin species were found to reveal almost identical structural features like those following from X-ray diffraction files. The difference between DFT results and the respective real crystal values amounted only $1.0 \%-2.3 \%$ (Table 2 ), which practically justifies the significance of molecular calculations. Although the DFT-calculated structures have been referred to vacuum conditions, the general features shown by the derived molecular models, such as deviations from core-planarity, matched well those observed in real crystals. For details concerning the calculated Zn-porphyrin structures see the Supplementary information appendix. Moreover, as follows from Table 2, the core dimensions in meso-substituted porphyrins do not differ from those determined for $\mathrm{Zn}$-porphine $(\mathrm{R}=\mathrm{H}$, Figure 1). This may indicate that the meso-substituents by no means do weaken the macrocycle's strength. Incidentally, the almost equal $\mathrm{C}_{\alpha}-\mathrm{N}$ and $\mathrm{C}_{\alpha}-\mathrm{C}_{\mu}$ bond lengths within the porphyrin core provide important evidence for a well organized and flexible bonding set-up and hence a very stable molecular system in tetra-meso-functionalized Zn-porphyrins. Thus, one may expect them to exhibit a considerable potential to prevent the porphyrin unit from degradation under unfavorable conditions. 
Indeed, the studied ZnTBPP complex appeared thermally resistant up to almost $540{ }^{\circ} \mathrm{C}$ (as determined from thermal analysis; see Experimental section). On the other hand the elastic molecular structure allows accommodating the energy of possible structural distortions which may determine the chemical activity of diverse zinc porphyrins, which appears particularly important in porphyrin-based catalysts.

\subsection{Crystal Surface Structure}

To supplement the above considerations dedicated principally to the molecular structure, the morphology of solid crystals has been briefly addressed, too. Scanning electron microscopy (SEM) investigations performed for the model ZnTBPP compound proved the crystals were wrinkled and porous, as shown in Figure 3. Most probably the solid's interior consists of a network of fine pores, creating a "sponge-like" structure. This feature seems to be common for a large number of diverse phenyl-meso-substituted metalloporphyrin analogues, so-called "porphyrin-sponges" [9,22,25]. Presumably, this is also the reason for a rather fragile construction of single crystals and the problems met while trying to grow large mono-crystals suitable for X-Ray measurements. On the other hand, however, such well-developed microstructure should offer enhanced sorption activity, particularly important in catalytic systems. Moreover, the studied material revealed surface-adsorbed oxygen, as determined by the X-ray microprobe analysis (EDAX). This finding has not been surprising however, and results from the peculiar affinity of porphyrin analogues to molecular oxygen [29].

Figure 3. SEM micrographs of ZnTBPP crystals. (a) $\times 500$; (b) $\times 10,000$ (enlargement of arrow-pointed area).

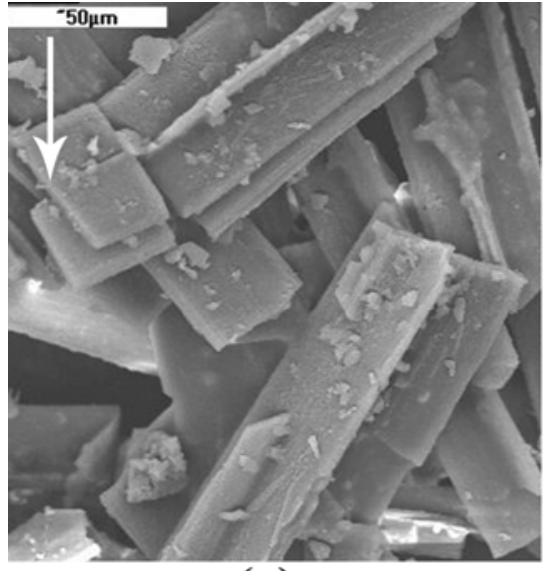

(a)

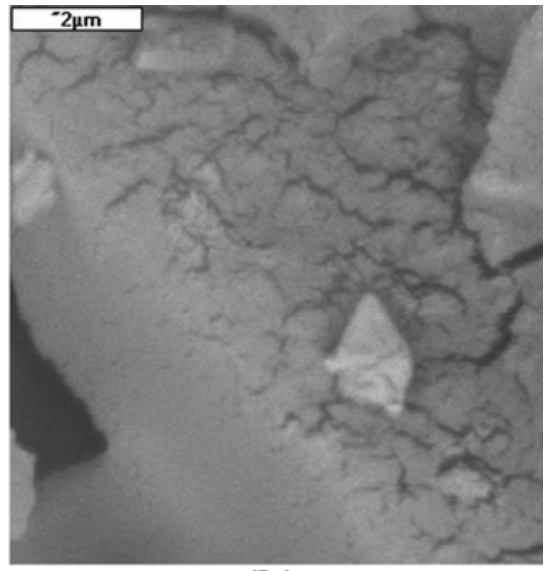

(b)

\subsection{Infrared Spectra and Structure-Related Effects Based on DFT Calculations}

External groups attached at the meso-carbon atom of the porphyrin core might have been suspected to induce some changes in electronic density distribution over the $C_{\alpha}-C_{\mu}-C_{\alpha}$ bridges, which eventually could have somehow affected the quality of these particular bonds. Although the structural analysis did not indicate for significant alterations in bond length, however the dynamic behavior of the macrocyclic bonding system could not be fully evaluated when exclusively based on crystallographic and theoretical molecular data. Therefore, the frozen-structure-related part of this work was supported 
by an infrared vibrational study to get some more profound insight into the porphyrin system modified by its functionalization at the bridging carbons $\left(\mathrm{C}_{\mu}\right)$.

The impact of the meso-substituents on the IR oscillations of bonds constituting the porphyrin core was examined for the model ZnTBPP compound and the selected set of diverse analogues shown in Figure 1. The particular active vibrational modes were identified based on DFT molecular calculations and using the animation feature of the GaussView 5.0 software. Besides, the FTIR spectrum measured for ZnTBPP was confronted with the DFT-simulated one (Figure 4). Additionally, the spectra calculated for Zn-porphine $(\mathrm{R}=\mathrm{H})$, tetraphenyl $(\mathrm{R}=1, \mathrm{X}=\mathrm{H})$ and tetrahydroxyphenyl $(\mathrm{R}=1, \mathrm{X}=\mathrm{OH})$ derivatives were compared with experimental FTIR data reported elsewhere, [14,30,31], respectively (see Supplementary Information appendix).

Figure 4. Experimental FTIR spectrum of ZnTBPP and the simulated IR spectrum obtained from DFT calculations.

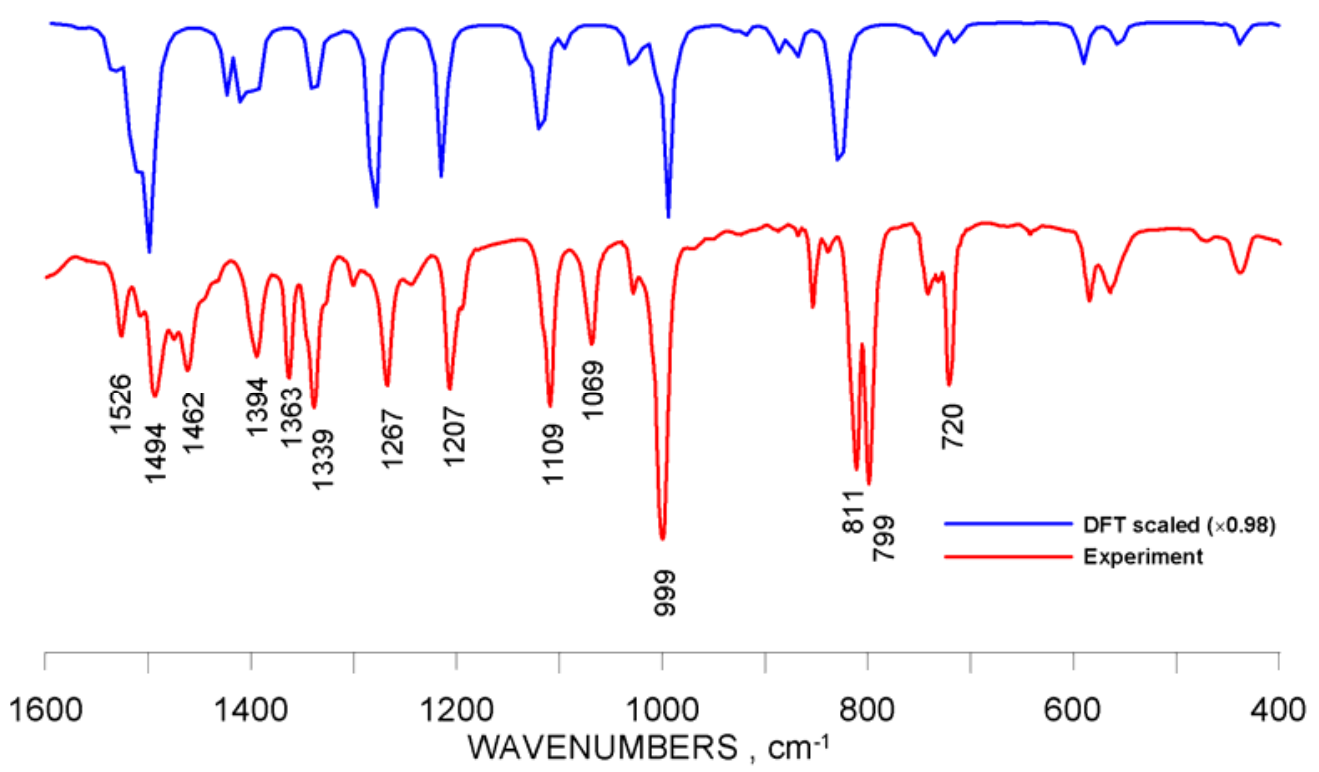

Comparison of the DFT-calculated spectra with the experimental results obtained both in this work and reported elsewhere basically indicates for common features of the spectrum patterns, although the peak values (frequency/intensity) in some cases differ from each other. Nevertheless, as shown in Figure 4, the scaled theoretical and real-measurement frequency values seem close enough to consider the DFT-derived IR spectral data important in advanced molecular studies. This assumption has found a strong backup in the relevant papers [12,13,32].

The oscillating porphyrin macrocycle actually represents a system of mutually coupled vibrations. Hence it is rather difficult to assign the frequency of the particular bands identified in the IR spectra to a localized oscillation mode. It proved possible (to a reasonable extent) extracting from the oscillating $\pi$-electronic setup those vibrations which refer to stretching of the $C_{\alpha}-C_{\mu}-C_{\alpha}$ bridges and these vibrations were used in further considerations. More tentatively however, the pure stretching vibrations of the $C_{\mu}-R$ bonds could be separated from other oscillations manifested by the functional group (R). The computed frequencies related to the above-mentioned vibrations have been reported in Table 3 . 
Table 3. Calculated frequencies $(v)$ of IR stretching vibrations (not scaled) assigned to the $\mathrm{C}_{\alpha}-\mathrm{C}_{\mu}-\mathrm{C}_{\alpha}$ bridges and (tentatively) to $\mathrm{C}_{\mu}-\mathrm{R}$ oscillations for diverse meso-substituted Zn-porphyrins (according to Figure 1). The first-row frequencies (bold italic font) apply to coupled diverse vibration modes, $\mathrm{C}_{\alpha}-\mathrm{C}_{\mu}$ and $\mathrm{C}_{\beta}-\mathrm{C}_{\beta}$ (see also Table SI-1, Supplementary Information appendix).

\begin{tabular}{|c|c|c|c|c|c|c|c|c|c|}
\hline \multicolumn{10}{|c|}{$\mathrm{v}, \mathrm{cm}^{-1}$} \\
\hline \multirow{2}{*}{$\begin{array}{c}\text { Oscillation } \\
\text { mode }\end{array}$} & \multirow{2}{*}{$\mathbf{R}=\mathbf{H}$} & \multicolumn{6}{|c|}{$R=1$ and $X$ as below } & \multirow{2}{*}{$\mathbf{R}=\mathbf{2}$} & \multirow{2}{*}{$\mathbf{R}=\mathbf{3}$} \\
\hline & & H & $\mathbf{F}$ & $\mathbf{C N}$ & OH & OMe & $\mathbf{t B u}$ & & \\
\hline \multirow{3}{*}{$\mathrm{C}_{\alpha}-\mathrm{C}_{\mu}$} & & 1565 & 1566 & 1568 & 1565 & 1565 & 1565 & \multirow{3}{*}{$\begin{array}{l}1575 \\
1533\end{array}$} & \multirow{3}{*}{$\begin{array}{l}1562 \\
1538\end{array}$} \\
\hline & $\begin{array}{l}1537 \\
1507\end{array}$ & 1539 & 1544 & 1546 & 1547 & 1546 & 1549 & & \\
\hline & & 1520 & 1527 & 1531 & 1529 & 1529 & 1531 & & \\
\hline \multirow{2}{*}{$C_{\mu}-R$} & 3204 & 1648 & 1649 & 1655 & 1661 & 1658 & 1658 & 1414 & 1603 \\
\hline & & 1254 & 1253 & 1254 & 1254 & 1254 & 1258 & 1179 & 1191 \\
\hline
\end{tabular}

The effect of the diverse meso-substituent type on the character of the IR spectra was illustrated in Figure 5a. On the other hand, the influence of the phenyl-derivatives most frequently used to substitute the meso-carbons of the porphyrin moiety was shown in Figure 5b. Full-range IR spectra derived from DFT theoretical calculations along with a listing (Table SI-1) of oscillation frequencies for vibrations basically related to the porphyrin macrocycle have been presented in the Supplementary information appendix.

Figure 5. Infrared spectra calculated for selected Zn-porphyrins (raw results, not scaled). (a) Effect of the meso-substituent type. General molecular structure is shown; (b) Effect of the X-group in phenyl-meso-substituents.

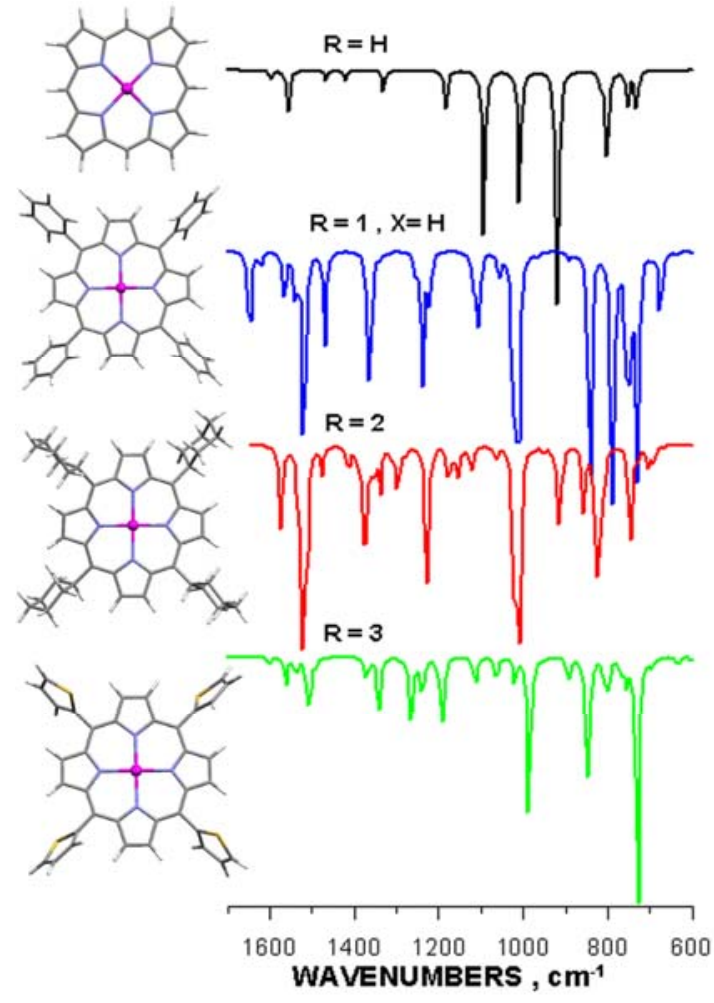

(a)

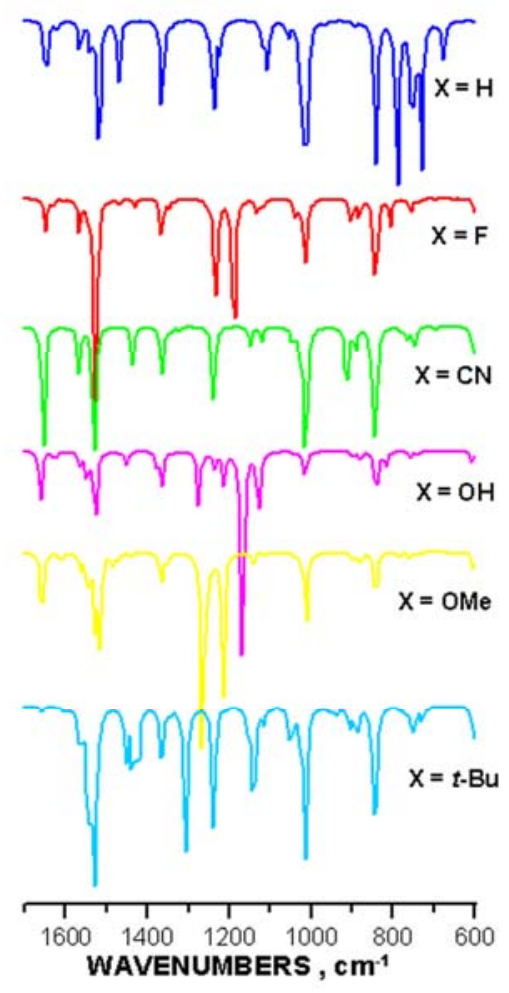

(b) 
In principle, the IR spectra conform very well to the crystallographic data analyzed in the preceding section and do supplement the conclusions drawn from structural considerations. Common features observed in the spectra displayed in Figure 5a essentially apply to the pyrrole unit of the macrocycle and may be assigned to the stretching of the $\mathrm{C}_{\beta}-\mathrm{C}_{\beta}$ bonds $\left(1557-1575 \mathrm{~cm}^{-1}\right)$, "pyrrole-breathing" oscillations (988-1018 $\left.\mathrm{cm}^{-1}\right)$ and $\mathrm{C}_{\beta}-\mathrm{H}$ in plane $\left(1330-1370 \mathrm{~cm}^{-1}\right)$ and/or out-of-plane $\left(820-850 \mathrm{~cm}^{-1}\right)$ vibrations (Table SI-1). On the other hand, the most apparent differences, which one may see in the spectra, follow from oscillations of the bonding system of the meso-substituents, R (see also the fullrange spectra in Supplementary information). However, a closer look at the spectra has revealed some details evidently reflecting the impact of the functional group on the macrocycle's electronic system, due to different molecular volume and chemistry of the particular substituents (Table 3). Surely, the subtle distinction in frequency of the pyrrole-related oscillations one may have linked with the nature of the species attached at the meso-C atoms. The stretching vibrations of the $\mathrm{C}_{\alpha}-\mathrm{C}_{\mu}$ bonds characterize the possible electronic distribution in the vicinity of the meso-carbons, considered the most "sensitive" part of the porphyrin core and therefore, to some extent, the flexibility and strength of the macrocycle. Relatively similar values obtained for the phenyl-, cyclohexyl- and thienyl- analogues suggest that these groups induce a comparable molecular effect upon the porphyrin core. Only the frequency of $\mathrm{C}_{\alpha}-\mathrm{C}_{\mu}$ vibrations in the case of $\mathrm{Zn}$-porphine $(\mathrm{R}=\mathrm{H})$ stands out of the row (Table 3), thus probably reflecting the lack of meso-substituted carbons. Conversely, stretching vibrations of the $\mathrm{C}_{\mu}-\mathrm{R}$ bonds are featured by very different frequencies depending on the type of R. Definitely the least values were demonstrated by the cyclohexyl-derivative, which probably indicates for a weaker $\mathrm{C}_{\mu}-\mathrm{R}$ connection. Obviously, the $\mathrm{C}_{\mu}-\mathrm{H}$ stretching was found to be far out of the range determined for the bulky substituents $\left(R=\mathbf{1}, 2\right.$ and 3), at $3204 \mathrm{~cm}^{-1}$. Besides, the oscillation mode of the $C_{\mu}-R$ bonds proved to follow the vibrations observed for the functional group itself. Therefore the frequencies assigned to such seemingly localized vibrations are supposed to be treated with particular caution. In fact, they might have been reflecting rather the nature of the oscillating bonding system of the meso-substituent and not that of the $\mathrm{C}_{\mu}-\mathrm{R}$ bond.

The phenyl-substituted porphyrins $(\mathrm{R}=\mathbf{1}, \mathrm{X}$, Figure 1$)$ display practically very similar spectral features in the part related merely to the macrocycle, as follows from Figure $5 \mathrm{~b}$ and Table 3 . Almost equal frequencies of the $\mathrm{C}_{\alpha}-\mathrm{C}_{\mu}$ vibrational modes as well as of those assigned to the pyrrole unit suggest the $\pi$-electronic system of the porphyrin core is not affected by the peripheral $\mathrm{X}$ substituents. The same conclusion applies when regarding the $\mathrm{C}_{\mu}$-R stretching vibrations. Presumably, there might be some very subtle infrared-sensitive core-effects generated by the diverse phenyl-substituents, however these are of little importance and usually are not to be seen in the relevant IR spectra due to low intensity of such oscillations.

\section{Experimental}

\subsection{Materials}

5,10,15,20-Tetrakis(4-tert-butylphenyl)porphyrinato zinc(II) (ZnTBPP) was synthesized according to a typical procedure reported elsewhere [5], using $\mathrm{ZnCl}_{2}$ as the metal source. Pure monocrystals for crystallographic measurements were obtained by multiple re-crystallization from toluene (reagent 
grade) at room temperature, yielding the final product in the form of a toluene solvate. This complex was used in this study as a reference model zinc porphyrin and it has been characterized as follows: UV-vis (toluene): $\lambda_{\max }, \mathrm{nm}(\log \varepsilon) 425$ (5.40), 551 (4.05), 591 (3.36); FT-IR (KBr): $v, \mathrm{~cm}^{-1} 3029$ (w), 2960 (s), 2903 (m), 2867 (m), 1526 (w), 1496 (m), 1462 (m), 1394 (m), 1363 (m), 1339 (m), 1267 (m), 1207 (m), 1109 (m), 1069 (m), 999 (vs), 811 (s), 799 (s), 720 (m); ${ }^{1} \mathrm{H}-\mathrm{NMR}$ (400 MHz; CDCl ${ }_{3}$; $\left.\mathrm{Me}_{4} \mathrm{Si}\right): \delta_{\mathrm{H}}, \mathrm{ppm} 1.62\left(36 \mathrm{H}, \mathrm{s},-\mathrm{CH}_{3}\right), 7.76(8 \mathrm{H}, \mathrm{d}, \mathrm{Ph}), 8.15(8 \mathrm{H}, \mathrm{d}, \mathrm{Ph}), 8.98(8 \mathrm{H}, \mathrm{s}$, pyrrole- $H)$; ${ }^{13} \mathrm{C}-\mathrm{NMR}$ (400 MHz; $\mathrm{CDCl}_{3} ; \mathrm{Me}_{4} \mathrm{Si}$ ): $\delta$, ppm 29.66, 31.68, 121.11, 123.39, 131.90, 129.00, 134.28, 139.81, 150.26; thermal analysis (DSC): endoergic peak at $538.9{ }^{\circ} \mathrm{C}$, DTG peak at $537.4{ }^{\circ} \mathrm{C}(92 \%$ loss of mass) corresponding to the compound decomposition. Electronic absorption and emission spectra (UV-Vis), ${ }^{1} \mathrm{H}-\mathrm{NMR}$ and FTIR raw spectra have been included in the supplementary information appendix.

\subsection{Computational Methods}

Density functional theory (DFT) method was applied to calculate the molecular structures and infrared spectra of selected porphyrins (Figure 1). The appropriate Z-matrices have been provided in the supporting information appendix. All calculations were performed using the Gaussian 09 software [20]. Geometry optimizations and frequency analysis for all studied compounds were carried out using the hybrid density functional B3LYP [33,34] and the LANL2DZ basis set [35]. The B3LYP functional has proved remarkable accuracy in many real-world problems [36,37] and has been considered probably the best compromise between speed and accuracy for relatively large systems such as functionalized metalloporphyrins or metallophthalocyanines. This was demonstrated in our recently published works $[8,15]$.

\subsection{Instrumental Methods}

\subsubsection{X-ray Powder Diffractometry}

Diffraction pattern of the pulverized polycrystalline material, ZnTBPP, was recorded on a Philips PW1710 diffractometer using $\mathrm{CuK}_{\alpha}$ radiation $\left(\lambda_{\alpha 1}=1.54060 ; \lambda_{\alpha 2}=1.54439 \AA \hat{)}\right)$. Complete set of powder XRD data is provided as supplementary material (see Electronic Supplementary data).

\subsubsection{Scanning Electron Microscopy (SEM)}

Investigations were carried out by a JEOL JSM-5400 scanning electron microscope provided with an energy dispersive X-ray analyzer (EDAX). Prior to testing, the samples were evacuated (298 K, $100 \mathrm{~Pa})$ and gold coated.

\subsubsection{Electronic Absorption Spectroscopy}

UV-VIS spectra were measured by the means of a UNICAM 310 spectrophotometer within the range of $190-1100 \mathrm{~nm}$, in a $10 \mathrm{~mm}$ quartz cuvette, at constant temperature $20^{\circ} \mathrm{C}$ (thermostated). 


\subsubsection{Electronic Emission Spectroscopy (Fluorescence)}

Fluorescence spectra were recorded in toluene for $\lambda_{\text {exc }}=360 \mathrm{~nm}$, using Perkin-Elmer MPF-3 spectrofluorimeter. Fluorescence quantum yield, $\Phi_{\mathrm{F}}$, was determined by a comparative method using $\left[\mathrm{Ru}(\mathrm{bpy})_{3}\right] \mathrm{Cl}_{2}$ as standard.

\subsubsection{FTIR Spectroscopy}

Philips FTIR PU 9800 spectrometer was used to measure the FTIR spectrum and the zinc porphyrin sample (ZnTBPP) was analyzed in a $\mathrm{KBr}$ disc.

\subsubsection{NMR Spectroscopy}

${ }^{1} \mathrm{H}-\mathrm{NMR}$ and ${ }^{13} \mathrm{C}-\mathrm{NMR}$ spectra were recorded on a Bruker Avance II $400 \mathrm{~Hz}$ spectrometer.

\subsubsection{Thermal Analysis (DSC, DTG)}

Universal V3.0G device (TA Instruments) was used to register the DSC (Differential Scanning Calorimetry) and TGA (Thermogravimetry Analysis) runs, under constant nitrogen flow (40 $\mathrm{mL} \mathrm{N} / 2 \mathrm{~min})$ and at a heating rate of $10 \mathrm{deg} / \mathrm{min}$.

\section{Conclusions}

The bonding system in tetra-meso-substituted zinc porphyrins constitutes a very stable and flexible molecular set-up. Both the crystallographic and IR investigations have indicted that the considered meso-substituents practically do not affect the strength of the porphyrin core. DFT theoretical investigations appeared very useful in analyzing both structural and IR-spectral properties. It must be emphasized, that the IR spectra reported in this work well conform to the quoted references concerning both the experimental data and theoretically derived IR vibrational frequencies. Diverse structural effects observed in real Zn-porphyrin crystals may appear crucial to the catalytic activity of such compounds. The flexible porphyrin core allows the molecule to accumulate extra energy resulting from intermolecular interactions, which could even produce structural deformations but not disruption of the bonding system. Thus, under certain circumstances one may expect such compounds to exhibit enhanced reactivity. Therefore structural investigations are of principal meaning particularly in molecular designing of porphyrin solids considered for application in heterogeneous systems.

\section{Supplementary Material}

Supplementary material can be accessed on: http://www.mdpi.com/1420-3049/16/12/9957/s1.

\section{Acknowledgements}

Theoretical DFT calculations were supported by the grant obtained from the Academic Computer Center CYFRONET AGH Kraków, no. KBN/SGI2800/UOpolski/012/2001. 


\section{References and Notes}

1. Kadish, K.M.; Smith, K.M.; Guilard, R. Handbook of Porphyrin Science; World Scientific Publishing Co.: Singapore, 2010-2011.

2. Mele, G.; Del Sole, R.; Vasapollo, G.; García-López, E.; Palmisano, L.; Li, J.; Słota, R.; Dyrda, G. $\mathrm{TiO}_{2}$-based photocatalysts impregnated with metallo-porphyrins employed for degradation of 4-nitrophenol in aqueous solutions: Role of metal and macrocycle. Res. Chem. Intermed. 2007, 33, 433-448.

3. Wei, X.; Du, X.; Chen, D.; Chen, Z. Thermal analysis study of 5,10,15,20-tetrakis (methoxyphenyl) porphyrins and their nickel complexes. Thermochim. Acta 2006, 440, 181-187.

4. Lebedeva, N.S.; Yakubov, S.P.; Vyugin, A.I.; Parfenyuk, E.V. Thermodynamics of complex formation of natural iron(III)porphyrins with neutral ligands. Thermochim. Acta 2003, 404, 19-24.

5. Mele, G.; Del Sole, R.; Vasapollo, G.; García-López, E.; Palmisano, L.; Schiavello, M.J. Photocatalytic degradation of 4-nitrophenol in aqueous suspension by using polycrystalline TiO2 impregnated with functionalized $\mathrm{Cu}(\mathrm{II})$-porphyrin or $\mathrm{Cu}(\mathrm{II})$-phthalocyanine. J. Catal. 2003, 217, 334-342.

6. Słota, R.; Dyrda, G.; Szczegot, K.; Mele, G.; Pio, I. Photocatalytic activity of nano and micro crystalline $\mathrm{TiO}_{2}$ hybrid systems involving phthalocyanine or porphyrin sensitizers. Photochem. Photobiol. Sci. 2011, 10, 361-366.

7. Słota, R.; Mele, G.; Ejsmont, K.; Domański, A.A.; Del Sole, R. [5,10,15,20-Tetrakis(4-tertbutylphenyl)porphyrinato- $\left.\kappa^{4} N\right]$ zinc(II) toluene solvate. Acta Cryst. E 2007, 63, m2582.

8. Man, D.; Słota, R.; Broda, M.A.; Mele, G.; Li, J. Metalloporphyrin intercalation in liposome membranes: ESR study. J. Biol. Inorg. Chem. 2011, 16, 173-181.

9. Kumar, R.K.; Balasubramanian, S.; Goldberg, I. Porphyrin sponges: Structural systematics of the host lattice. Inorg. Chem. 1998, 37, 541-552.

10. Veyrat, M.; Ramasseul, R.; Turowska-Tyrk, I.; Scheidt, W.R.; Autret, M.; Kadish, K.M.; Marchon, J.-C. Nickel(II) and zinc(II) meso-tetracyclohexylporphyrins. Structural and electronic effects induced by meso-cyclohexyl substitution in metalloporphyrins. Inorg. Chem. 1999, 38, 1772-1779.

11. Purushothaman, B.; Varghese, B.; Bhyrappa, P. [5,10,15,20-Tetrakis(2-thienyl)-porphyrinato] zinc(II). Acta Cryst. C 2001, 57, 252-253.

12. Yao, P.; Han, S.; Zhang, Y.; Zhang, X.; Jiang, J. Structures and spectroscopic properties of mesotetrasubstituted porphyrin complexes: meso-Substitutional and central metallic effect study based on density functional theory calculations. Vib. Spectrosc. 2009, 50, 169-177.

13. Zhang, Y.-H.; Zhao, W.; Jiang, P.; Zhang, L.-J.; Zhang, T.; Wang, J. Structural parameters and vibrational spectra of a series of zinc meso-phenylporphyrins: A DFT and experimental study. Spectrochim. Acta A 2010, 75, 880-890.

14. Oliveira de, V.E.; Cimini Correa, C.; Pinheiro, C.B.; Diniz, R.; Oliveira de, L.F.C. Structural and spectroscopy studies of the zinc complex of p-hydroxyphenylporphyrin. J. Mol. Struct. 2011, 995, 125-129. 
15. Wang, C.; Yang, G.; Li, J.; Mele, G.; Słota, R.; Broda, M.A.; Duan, M.; Vasapollo, G.; Zhang, X.; Zhang, F.X. Novel meso-substituted porphyrins: Synthesis, characterization and photocatalytic activity of their $\mathrm{TiO}_{2}$-based composites. Dyes Pigments 2009, 80, 321-328.

16. Allen, F.H. The Cambridge Structural Database: A quarter of a million crystal structures and rising. Acta Cryst. B 2002, 58, 380-388.

17. Van de Streek, J. Searching the Cambridge Structural Database for the best representative of each unique polymorph. Acta Cryst. B 2006, 62, 567-579.

18. Orpen, A.G. Applications of the Cambridge Structural Database to molecular inorganic chemistry. Acta Cryst. B 2002, 58, 398-406.

19. Allen, F.H.; Motherwell, W.D.S. Applications of the Cambridge Structural Database in organic chemistry and crystal chemistry. Acta Cryst. B 2002, 58, 407-422.

20. Frisch, M.J.; Trucks, G.W.; Schlegel, H.B.; Scuseria, G.E.; Robb, M.A.; Cheeseman, J.R.; Scalmani, G.; Barone, V.; Mennucci, B.; Petersson, G.A.; et al. Gaussian 09, Revision A.02; Gaussian, Inc.: Wallingford, CT, USA, 2009.

21. Veyrat, M.; Ramasseul, R.; Marchon, J.-C.; Turowska-Tyrk, I.; Scheidt, W.R. Nickel(II) and zinc(II) complexes of meso-tetrakis(cyclohexyl)porphyrin: Distinct types of porphyrin distortion in response to steric crowding. New J. Chem. 1995, 19, 1199-1202.

22. Byrn, M.P.; Curtis, C.J.; Hsiou, Y.; Khan, S.I.; Sawin, P.A.; Tendick, S.K.; Terzis, A.; Strouse, C.E. Porphyrin sponges: Conservation of host structure in over 200 porphyrin-based lattice clathrates. J. Am. Chem. Soc. 1993, 115, 9480-9497.

23. Krupitsky, H.; Stein, Z.; Goldberg, I. Structural patterns in clathrates and crystalline complexes of zinc-tetra(4-chlorophenyl)porphyrin and zinc-tetra(4-fluorophenyl)porphyrin. J. Incl. Phenom. Macro. Chem. 1995, 20, 211-232.

24. Goldberg, I.; Krupitsky, H.; Stein, Z.; Hsiou, Y.; Strouse, C.E. Supramolecular architectures of functionalized tetraphenylmetalloporphyrins in crystalline solids. Studies of the 4-methoxyphenyl, 4-hydroxyphenyl and 4-chlorophenyl derivatives. Supramol. Chem. 1994, 4, 203-221.

25. Byrn, M.P.; Curtis, C.J.; Goldberg, I.; Hsiou, Y.; Khan, S.I.; Sawin, P.A.; Tendick, S.K.; Strouse, C.E. Porphyrin sponges: Structural systematics of the host lattice. J. Am. Chem. Soc. 1991, 113, 6549-6557.

26. Dastidar, P.; Krupitsky, H.; Stein, Z.; Goldberg, I. Crystal inclusion chemistry of zinc-tetra(4bromophenyl) porphyrin. J. Incl. Phenom. Macro. Chem. 1996, 24, 241-262.

27. Chiaroni, A.; Riche, C.; Bied-Charreton, C.; Dubois, J.C. Structure de la tetra(p-n-octylphenyl)5,10,15,20 porphine de zinc(II). Acta Cryst. C1988, 44, 429-432.

28. Golder, A.J.; Nolan, K.B.; Povey, D.C.; Milgrom, L.R. The structure of the palladium(II) and zinc(II) complexes of $\alpha, \beta, \gamma, \delta$-tetrakis(3,5-di-tert-butyl-4-hydroxyphenyl)porphyrin. Acta Cryst. C 1988, 44, 1916-1921.

29. Słota, R.; Dyrda, G.; Hnatejko, Z.; Karolczak, J.; Stryła, Z. Effect of air absorbed oxygen and moisture on the chemical stability of photoexcited phthalocyanines in dimethylformamide. J. Porphyr. Phthalocya. 2006, 10, 43-54.

30. Jarzęcki, A.A.; Kozłowski, P.M.; Pulay, P.; Ye, B.-H.; Li, X.-Y. Scaled quantum mechanical and experimental vibrational spectra of magnesium and zinc porphyrins. Spectrochim. Acta A 1997, 53, 1195-1209. 
31. Andersson, L.A.; Loehr, T.M.; Thompson, R.G.; Strauss, S.H. Influence of symmetry on the vibrational spectra of $\mathrm{Zn}(\mathrm{TPP}), \mathrm{Zn}(\mathrm{TPC})$, and $\mathrm{Zn}$ (TPiBC). Inorg. Chem. 1990, 29, 2142-2147.

32. Kozlowski, P.M.; Bingham, J.R.; Jarzecki, A.A. Theoretical analysis of core size effect in metalloporphyrins. J. Phys. Chem. A 2008, 112, 12781-12788.

33. Becke, A.D. Density functional thermochemistry. III. The role of exact exchange. J. Chem. Phys. 1993, 98, 5648-5652.

34. Lee, C.; Yang, W.; Parr, R.G. Development of the Colle-Salvetti correlation-energy formula into a functional of the electron density. Phys. Rev. B 1988, 37, 785-789.

35. Hay, P.J.; Wadt, W.R. Ab initio effective core potentials for molecular calculations. Potentials for the transition metal atoms Sc to Hg. J. Chem. Phys. 1985, 82, 270-283.

36. Singleton, D.A.; Wang, Z.H. Isotope effects and the nature of enantioselectivity in the shi epoxidation. The Importance of asynchronicity. J. Am. Chem. Soc. 2005, 127, 6679-6685.

37. Schneebeli, S.T.; Hall, M.L.; Breslow, R.; Friesner, R. Quantitative DFT modeling of the enantiomeric excess for dioxirane-catalyzed epoxidations. J. Am. Chem. Soc. 2009, 131, 3965-3973.

Sample Availability: Samples of ZnTBPP are available from the authors.

(C) 2011 by the authors; licensee MDPI, Basel, Switzerland. This article is an open access article distributed under the terms and conditions of the Creative Commons Attribution license (http://creativecommons.org/licenses/by/3.0/). 